\title{
China's Mental Health Interventions During the COVID-19 Outbreak
}

\author{
Zhengkui Liu ${ }^{\mathrm{a}^{*}}$, Yuanyuan $\mathrm{An}^{\mathrm{b}}$, Kankan $\mathrm{Wu}^{\mathrm{a}, \mathrm{c}}$ \\ ${ }^{a}$ CAS Key Laboratory of Mental Health, Institute of Psychology, Beijing, China \\ ${ }^{\mathrm{b}}$ School of Psychology, Nanjing Normal University, Nanjing, China \\ ${ }^{c}$ Department of Psychology, University of Chinese Academy of Sciences, Beijing, China
}

${ }^{\star}$ Corresponding author. E-mail: liuzk@psych.ac.cn

Background. The COVID-19 outbreak has threatened both the physical health of individuals who contracted the virus, and the mental health of everyone directly or indirectly associated with or concerned about it.

Objective. As telecommunication technologies and online mental health apps become more available and affordable, they allow behavioral and mental health professionals to provide quality care by handling problems arising from the COVID-19 outbreak virtually. The aim of the current article is to summarize the online psychological assistance supported by the Chinese government during the epidemic.

Design. Several measures, policies, action plans, and programs that have been underway in China during the COVID-19 outbreak epidemic are listed to provide guidance for mental health intervention practices around the world.

Results. A total of seven types of mental health services and supports developed in China were listed and introduced: 1) online psychological assistance; 2) online psychological self-assessment and self-help; 3) a "Peace of Mind" selfhelp counseling camp; 4) a "Peace of Mind" self-help training camp; 5) mental health training and lectures; 6) psychological assistance to Hubei; and 7) collaboration with social workers in "Thousands of Institutions Send Peace of Mind." Moreover, several areas for the organization and management of psychological intervention activities in the future were identified.

Conclusion. Mental health interventions helped people cope with their mental health concerns during the outbreak of COVID-19. They could facilitate the development of Chinese public emergency interventions, and eventually improve the quality and effectiveness of emergency interventions in China.

Keywords:

COVID-19;

China; mental health; emergency interventions; psychological assistance 


\section{Introduction}

The COVID-19 outbreak, first reported in China in late 2019, has shown up all around the world in 2020. Based on reports from China, where the outbreak was initially reported, the epidemic not only threatens the physical health of those who contract the virus, but also seriously affects the mental health of everyone directly or indirectly associated with or concerned about it.

In China, the national standards on mental health services, especially psychological counseling and crisis intervention, were established after the Wenchuan earthquake in 2008 (Zhang et al., 2012). The emergency medical rescue efforts after the earthquake, and lessons learned from them, quickly became important mechanisms guiding the public health field in addressing psychological issues in the population (Duan, \& Zhu, 2020).

Encouraged by the emphasis placed by the president of China on strengthening psychological intervention and humanistic care, and guided by the measures and emergency policies taken by the Chinese government for psychological assistance, the Chinese Psychological Society (CPS), together with professional psychological workers, started working diligently to improve the mental health of the general public and the health practitioners during the COVID-19 epidemic. A list of the measures, policies, action plans, and programs that have been implemented in China since the pandemic are reported here, in hopes of providing guidance on mental health intervention practices around the world.

\section{Measures and Emergency Policies Taken by the Chinese Government for Psychological Assistance During the Epidemic}

On February 3, 2020 the president of the People's Republic of China, Xi Jinping, emphasized the need to strengthen psychological intervention and humanistic care. On February 23, Xi delivered an important speech at the Conference on the Overall Promotion of Economic and Social Development and Deployment of Prevention and Control of COVID-19 (Xinhuanet, 2020), where he put a particular emphasis on on "strengthening the psychological guidance and intervention of the public."

At the beginning of the outbreak, the National Health Commission of People's Republic of China issued guidelines for emergency psychological crisis intervention for people affected by COVID-19 (National Health Commission of China, 2020). Recently, they also published specific guidelines for mental health services (National Health Commission of China, 2020). The Working Committee of Clinical Psychology Registration of the Chinese Psychological Society (CPS) issued a letter of recommendation to all national clinical psychological registrants immediately following the outbreak, encouraging members to participate in the psychological assistance work during the pandemic period.

As telecommunication technologies and health apps have become more available and affordable, they have expanded opportunities for behavioral and mental health professionals to provide quality care (Luxton, Nelson, \& Maheu, 2016). As of February 9, there are almost 448 psychological assistance hotlines available either by phone or chat apps inside and outside Wuhan city. Among them are 11 telephone hotlines and eight psychological online support apps providing help mainly for medical workers in Wuhan (The Paper, 2020). Hotlines of psychological assis- 
tance have been utilized to offer customized counseling services on a one-on-one basis, following the notice on establishing the psychological assistance hotline for the epidemic issued by the Novel Coronavirus Infection Control and Joint Control Mechanism of the State Council (www.gov.cn, 2020).

Soon after professional psychological workers were recruited, the Chinese Psychological Society (CPS) officially launched the "Peace of Mind" programs against epidemic situations. The timeline of the "Peace of Mind" programs was tentatively set from January 2020 to January 2022, and it is divided into two stages: the emergency period and the post- epidemic psychological reconstruction period.

Due to the high infection rate and quarantine needs, traditional face-to-face psychological services cannot be offered. "Peace of mind" programs thus emphasize the application of internet and artificial intelligence technology in publicizing mental health knowledge and constructing psychological hotlines. There are six main types of services in this project.

\section{Online psychological assistance}

This type of service is offered through short-term online consultation and the WeChat hotline. The Chinese Psychological Society, the Alipay Public Welfare Foundation, Ali Health, and WeChat jointly built a new type of coronavirus national psychological online service platform and embarked on the online service on January 30. The network system can match consultants with clients based on a questionnaire filled out by the client. Up to now, the psychological counseling service on the Alipay platform has received more than 230,000 visits and carried out 6,773 counseling sessions.

\section{Online psychological self-assessment and self-help}

This type of service includes filling out questionnaires which assess levels of anxiety, depression, obsession, and sleep; cognitive behavioral therapy (including reading, self-guidance technology, and multimedia guidance) dealing with the abovementioned problems was provided following the assessment. As of February 3, after it was launched online, more than 4500 people participated in the assessment and completed the CBTi self-help adjustment. Another platform is an app targeting women's mental health. Games were devised to help with relieving negative emotions and pressures, and embedded in the app. This app was officially launched on February 8 and has been utilized by 71,877 people as of the writing of this report.

\section{3. "Peace of Mind" self-help counseling camp}

Given that the front-line medical staff and clinical psychologists in Wuhan are weighed down with heavy insulation garments, they cannot effectively carry out psychological decompression and counseling. The "Peace of Mind" self-help training camp invented the PM+ project of WHO (World Health Organization, 2016) and made it an online app which guides relaxation training, positive self-cognition training, and mindfulness training for anyone who is seeking social support, especially front-line medical staffs and patients in Wuhan. The training camp lasts for seven days and aims to help participants feel more connected and supported. 


\section{4. "Peace of Mind" self-help training camp}

The "Self-Help Peace of Mind Series Training Camp" is an important part of the "Peace of Mind in the Fight against the Epidemic" action initiated by the Institute of Psychology of the Chinese Academy of Sciences and the Chinese Psychological Society. It aims to provide scientific and effective online self-help for medical workers, patients, and the public affected by the epidemic. "Training Camp" has a personal (patient) version, a medical care version, a parent-child version, and a public version, each aimed at providing help for specific groups of users. The various versions are described below.

1) Self-Help Peace of Mind Training Camp (public version). The public version of the Self-Help Peace of Mind Training Camp provides the public with methods and courses for emotional adjustment, helping everyone increase their connections with others in a limited environment and increase their social support. As of now, a total of 25,702 people has participated, and 87,533 check-ins have been recorded.

2) Self-Help Peace of Mind Training Camp (parent-child version). The parentchild version of the Self-Help Peace of Mind Training Camp is organized around parent-child and family decompression, with 10-20 minutes of psychological support and counseling a day to accompany and support parents or caregivers in helping them to understand and take care of their children. The counseling aims to help children cope with the stress of the epidemic in a positive and stable state of mind and body, by making the family a spiritual haven for them. As of now, a total of 19,208 people has participated, and 40,747 check-ins have been recorded.

3) Self-Help Peace of Mind Training Camp (medical care version). The medical care version of the Self-Help Peace of Mind Training Camp targets frontline medical staff in response to infectious diseases outbreaks and other public health emergencies. It supports medical staff in taking care of themselves through a "psychological intervention with multi-technology integration," which is evidence-based, professional, and convenient. It also aids medical staff in relieving continuous and possible stress, and maintaining a good physical and mental state. So far, 963 people have participated, and 1,759 check-ins have been recorded.

4) Self-Help Peace of Mind Training Camp (personal version). The personal version of the Self-Help Peace of Mind Training Camp provides psychological support and self-help psychological counseling for patients with the COVID-19 virus. Up to now, 566 people have participated, and 1,373 check-ins have been recorded.

\section{Mental health training and lectures}

"Peace of Mind lectures" are broadcast live over the whole network. The program invites the most influential experts in the field of psychological crisis intervention in China to provide special lectures and training for all people affected by the epidemic, as well as the volunteers participating in psychological assistance for the fight against the epidemic, through a combination of live broadcast and record- 
ings. Multiple platforms have been launched, including Popular Science China, the Chinese Academy of Sciences, the China Science and Technology Museum, the All-China Women's Federation, the Chinese Academy of Sciences Mental Health Service Platform, the Psychological Institute of the Chinese Academy of Sciences Anti-epidemic Column, the Continuing Education Network, and Heart Education. So far, 129 lectures have been broadcast, and the official platform of "Peace of Mind" has accumulated 3,005,588 views.

\section{Psychological assistance to Hubei}

On March, Liu Zhengkui and three others formed a team. They went to Wuhan to carry out psychological assistance work, which was comprised of the following elements:

1. Participating in the analysis and assessment of the psychological assistance policy of the epidemic

Chen Xuefeng, Liu Zhengkui, Wu Kankan, and others visited the Wuhan Conference Center, where the National Health Commission is located, four times, to participate in the research and evaluation of the psychological assistance policy for the epidemic. They then drafted the "Work Plan for Psychological Counseling in the COVID-19 Epidemic," which was issued by the Joint Prevention and Control Mechanism of the State Council on March 18.

The team participated in three seminars organized by the Wuhan Municipal Committee's Political and Legal Committee on the construction of psychosocial services for the epidemic, and then submitted the "Wuhan Psychological Service under the Impact of the COVID-19 Epidemic Work Plan." They also participated in three seminars on psychological counseling and social work service plans organized by the Ministry of Civil Affairs, the Hubei Provincial Department of Civil Affairs, and the Wuhan Civil Affairs Bureau, to discuss the working mechanism of the heart-society linkage and the implementation of the "Five Communities, One Heart" program. They had four discussions on the formulation and promotion of psychological assistance programs with the Women's Federation of Wuchang District, Wuhan City, Hubei Province, and then established a weekly long-term communication mechanism to provide professional support for the Hubei Provincial Women's Federation in carrying out its pilot work.

2. Carrying out pilot psychological services in hospitals and communities

In terms of hospitals, the "Peace of Mind" program conducted investigations and provided services at the Jinyintan Hospital, the Wuhan Special Care Hospital, the Medical Team of the First Affiliated Hospital of the University of Science and Technology of China, and the Anhui Aid to Hubei Medical Team. "Peace of Mind" also provided medical staff with a psychological self-help program. In terms of recovery stations, it carried out investigations and provided services at four isolation points, including Qiaokou Xinhua Printing, Wuhan First Commercial School, the Yangtze River Engineering Vocational and Technical College, and Hubei University.

It also publicized psychological information to help recovered patients and staff at the isolation points, and provided self-help procedures and online and offline one-on-one psychological services. 
In terms of the community, it conducted investigations and provided services in the Kaiyuan Mansion Community and Jindi Garden Community in Qingshan District. It also provided psychological counseling for community officials, volunteers, and other staff. Self-help services were provided to residents. This program regularly visits the station at Hongshanfang, a pilot community of the Women's Federation of Hubei Province, to provide professional guidance to community psychologists.

3. Providing multi-technology integration of support for application services

"Peace of Mind" adopted a combination of online and offline methods for conducting extensive psychological research and service work during the epidemic. It distributed emotion-monitoring bracelets on-site in the lobby of the outpatient building of Jinyintan Hospital, to accumulate raw data and carry out scientific research while providing services for frontline medical staff and recovered patients who came for follow-up visits. It developed a variety of online mini-programs, online questionnaires, etc., and carried out epidemiological investigations and intervention studies for different groups of pregnant women, medical staff, and patients.

4. Providing psychological assistance for long-term positive patients

On April 20, a team led by Professor Shi Zhanbiao began to provide psychological assistance to 51 long-term positive patients with COVID-19 in Wuhan Jinyintan Hospital. The assistance includes multi-technology integrated psychological assistance, individual counseling, group counseling, and psychological science publications, among other services.

5. Providing psychological assistance for community residents

"Peace of Mind" joined with the Hubei Provincial Women's Federation, Wuchang District Women's Federation, Wuhan Huaxia Psychology, and others to establish a trinity team of community psychological service, which consisted of psychologists, psychological counselors, and community women's federation cadres in the Dongting, Bairuijing, and Hongshanfang communities in Wuchang District. This work was carried out in a fixed community in tour service mode one day per week; each household was provided psychological support twice.

The program also established a demonstration site called "Picture Book Home" in the Donghu Community, Liyuan Street, Hongshan District, where it provided 431 picture books and other books for children. It also provided 200 relief supply kits for children in need in the Hongshan, District, and Qingshan Districts, and presented one lecture on family psychological support for children affected by the epidemic in Wuhan. In addition, it administered a survey on the community residents' mental health situation. The mental health questionnaires of 1225 females and four mental health questionnaires for pregnant women residents were collected.

\section{Collaboration with social workers" "Thousands of Institutions Send Peace of Mind"}

On February 2, "Peace of Mind" action launched the "Thousands of Institutions Send Peace of Mind" project, which was guided by the Psychological Service Organization Working Committee of the Chinese Psychological Society, the Crisis Intervention Working Committee of the Chinese Psychological Society, and the 
Beautiful Community Program Office of the Chinese Social Work Federation. Under the overall planning of the National Psychological Service Organization Consortium, it has led thousands of social psychological service organizations, and social organizations to serve the key populations, communities, and families. Based on the communities, social organizations, and social workers, it assisted frontline workers and completed nine key public welfare psychological assistance tasks.

As of now, 590 organizations across the country have applied for this project, 520 organizations have undergone evaluation and review by the Executive Committee of "Peace of Mind," 390 psychosocial service organizations have been publicized, and 356 organizations have submitted work data feedback. The feedback data are as follows: 356 organizations have provided services to 2,576 designated groups, including 105,365 hotline services; 80,765 online questionnaires; 188,065 psychological assessments; 65,953 individual consultations; and 2,292,759 public welfare class services. The audio/video programs have recorded 3,541 visits, with 2,067,290 hits to play, and 5,794 popular science articles have been published, receiving $1,399,084$ hits to read.

\section{Conclusion}

In summary, various mental health services and supports are taking place to help citizens cope with mental health concerns due to the outbreak of COVID-19. They are facilitating the development of China's public emergency interventions and eventually could improve the quality and effectiveness of emergency interventions in China.

In addition to the aforementioned techniques, we identified several areas within the organization and management of psychological intervention activities to focus on in the future. The Wuhan Workstation of Psychological Assistance has become an important base for psychological services in response to the epidemic, and an important platform for the psychological service to complete 13 independent scientific research projects on psychological services for sufferers from the epidemic. The Workstation plans to carry out a two-year program of front-line psychological assistance and scientific research work. It will continue to systematically promote psychological services for epidemic prevention and control at three levels:

1. Promote the work of pilot hospitals and communities, refine service targets and research groups, and provide grief counseling for the families of the deceased, psychological counseling for the elderly, and psychological counseling for students returning to school. Provide psychological services to employees, family members, and students of the Wuhan branch system.

2. In response to special public health emergencies such as infectious diseases, focus on developing key technologies and network service platforms for epidemic prevention and control of "multi-technology-integrated psychological intervention"; coordinate with the mental health service platform of the Chinese Academy of Sciences; and explore psychological assistance with the support of the new model of "multi-technology integrated psychological intervention."

3. Strive to build a sound national emergency management system for major events, research the goals and ways of building such a psycho-social service system in China, and explore and promote its legalization, institutionalization, and stan- 
dardization. Provide frontline experience and knowledge which cannot be replaced by the national strategic scientific and technological power of the Psychological Institute, CAS and the Chinese Psychological Society (CPS).

\section{References}

Duan, L., \& Zhu, G. (2020). Psychological interventions for people affected by the COVID-19 epidemic. The Lancet Psychiatry, 7(4), 300-02. https://doi.org/10.1016/S2215-0366(20)30073-0

Luxton, D.D., Nelson, E.L., \& Maheu, M.M. (2016). A practitioner's guide to tele-mental health: How to conduct legal, ethical, and evidence-based telepractice. American Psychological Association.

National Health Commission of China (2020, January 27). A notice on the issuance of guidelines for emergency psychological crisis intervention in pneumonia for novel coronavirus infections. Retrieved from http://www.nhc.gov.cn/xcs/zhengcwj/202001/6adc08b966594253b2b791be5c 3b9467.shtml

National Health Commission of China (2020, March 5). A notice on strengthening psychological assistance and social work services in response to COVID-19. Retrieved from http://www.nhc.gov. $\mathrm{cn} / \mathrm{xcs} / \mathrm{zhengcwj} / 202003 / \mathrm{a} 9 \mathrm{~b} 0 \mathrm{bcb} 3 \mathrm{bb} 7445298 \mathrm{c} 480 \mathrm{c5003c51d6d.shtml}$

The Paper (2020, February 14). The National Health Commission of China has set up 448 free psychological assistance hotlines across the country. Retrieved from https://www.thepaper.cn/newsDetail_forward_5978673

World Health Organization. (2016). Problem management plus (PM+): individual psychological help for adults impaired by distress in communities exposed to adversity: WHO generic field-trial version 1.0 (No. WHO/MSD/MER/16.2). World Health Organization. Retrieved from https:// apps.who.int/iris/handle/10665/206417

www.gov.cn (2020, February 5). During the epidemic, 24-hour psychological assistance hotlines have been opened throughout the country. You can just call from here. Retrieved from http://www. gov.cn/fuwu/2020-02/05/content_5474792.htm

Xinhuanet (2020, February 23). Xi Jinping: Speech at the meeting on the coordinated promotion to prevent and control COVID-19 and the deployment of economic and social development. Retrieved from http://www.xinhuanet.com/politics/2020-02/23/c_1125616016.htm

Zhang, L., Liu, X., Li, Y., Liu, Y., Liu, Z., Lin, J., ... Liang, W. (2012). Emergency medical rescue efforts after a major earthquake: lessons from the 2008 Wenchuan earthquake. The Lancet, 379(9818), 853-861. Retrieved from https://doi.org/10.1016/S0140-6736(11)61876-X

Original manuscript received July 20, 2020

Revised manuscript accepted November 22, 2020

First published online December 30, 2020

To cite this article: Liu, Z., An, Yu., Wu, K. (2020). China's Mental Health Interventions During the COVID-19 Outbreak. Psychology in Russia: State of the Art, 13(4), 183-190. DOI: 10.11621/ pir.2020.0412 